\title{
Frakturendoprothetik des Azetabulums
}

\author{
Anna Janine Schreiner, Peter Marten de Zwart, Fabian Maria Stuby, Björn Gunnar Ochs
}

\section{Zusammenfassung}

Die Endoprothetik des Hüftgelenks bei traumatischen bzw. posttraumatischen Zuständen des Azetabulums betrifft ein kleines Patientenkollektiv. Der Zeitraum zwischen Verletzung und Hüfttotalendoprothese (H-TEP) nimmt mit steigendem Alter ab. Die endoprothetische Therapie umfasst neben der primären H-TEP-Implantation (ggf. mit additiver Osteosynthese) auch die sekundäre Implantation einer H-TEP, v. a. bei posttraumatischer Koxarthrose, und die verzögerte primäre Endoprothetik. Im primären Fall kommt zumeist ein Abstützring mit Bone Graft zur Verwendung und im sekundären Setting ein zementfreies Standardimplantat. Die Ergebnisse sind im Durchschnitt mit den Komplikationsraten und Langzeitergebnissen der Revisionsendoprothetik vergleichbar. Das OP-Team sollte sowohl Erfahrung in der Azetabulumchirurgie als auch in der Revisionsendoprothetik des Hüftgelenks haben.

\section{Fracture Endoprosthesis of the Acetabulum}

Total hip replacement (THR) after acetabular fracture or posttraumatic arthrosis of the acetabulum is only performed on a small group of patients. The period between trauma and THR decreases with increasing patient age. Apart from primary THR after acute acetabular fracture - if necessary with additional osteosynthesis -, therapy also includes secondary THR for posttraumatic arthrosis or delayed primary THR. For primary THR, a reconstruction ring is usually used in combination with bone graft and for secondary THR a regular cementless implant can be inserted. Results can usually be compared to those of revision THR. The team should be skilled in acetabular surgery as well as in revision THR.

\section{Einleitung}

Die Endoprothetik des Hüftgelenks bei traumatischen bzw. posttraumatischen Zuständen betrifft ein kleines Patientenkollektiv. Die Operationen sind sowohl im Fall der Versorgung einer Azetabulumfraktur als auch im Fall der primären bzw. sekundären Endoprothetik anspruchsvoll und bedürfen einer individuellen Indikationsstellung. Das OPTeam braucht daher Erfahrung in der Azetabulumchirurige wie auch in der Revisionsendoprothetik des Hüftgelenks. Insgesamt sind die Komplikationsraten

OP-JOURNAL 2016; 32: 196-205

(c) Georg Thieme Verlag KG Stuttgart · New York DOI http://dx.doi.org/10.1055/s-0042-121508 und Langzeitergebnisse der Frakturendoprothetik des Azetabulums vergleichbar mit denen der Hüftrevisionsendoprothetik.

\section{Epidemiologie Azetabulumfrakturen und posttraumatische Koxarthrose}

Mit einer Inzidenz von 3 Patienten/ 100000 Einwohner/Jahr zählen Azetabulumfrakturen zu den seltenen Verletzungen [1]. In der Hälfte der Fälle treten Azetabulumfrakturen im Rahmen eines Polytraumas auf [2]. Während bei jüngeren Patienten meist ein Hochrasanztrauma ursächlich ist [3], führen beim älteren Patienten häufig einfache Sturzereignisse zur Fraktur des Azetabulums [4]. Generell lässt sich sagen, dass ab dem 35. Lebensjahr die Rate an Niedrig- energietraumata als Unfallursache zunimmt [5]. Die demografische Entwicklung zeigt, dass die Fälle der jungen Patienten mit Hochrasanztrauma stagnieren, wohingegen die Patientengruppe mit der Altersfaktur des Azetabulums zunimmt [6] (Abb. 1).

In ca. $20 \%$ der Fälle tritt als häufigste Komplikation nach einer Azetabulumfraktur eine sekundäre bzw. posttraumatische Koxarthrose auf [7]. Eine weitere Komplikation stellen Femurkopfnekrosen dar, die im weiteren Verlauf ebenfalls der endoprothetischen Versorgung bedürfen können. Insgesamt sind ca. 12\% aller Koxarthrosen postraumatisch bedingt [8].

Komplikationen nach Azetabulumfraktur sind posttraumatische Koxarthrosen, Femurkopfnekrosen und Pseudarthrosen des Azebatulums.

\section{Pathomechanik der Azetabulumfraktur}

In der Regel führen Hochenergietraumata - z. B. im Rahmen von Verkehrsunfällen und Stürzen aus großer Höhe - zur Azetabulumfrakturen. Eine direkte azetabuläre Gewalteinwirkung ist selten und mit offenen Frakturen wie auch Weichteilverletzungen korreliert [9]. Letournel beschrieb die grundlegenden Mechanismen, die bei indirekter Gewalteinwirkung zur Azetabulumfraktur führen können. Der Frakturtyp hängt hierbei von der Stellung des Hüftkopfs in der Pfanne zum Krafteinleitungszeitpunkt ab. Im Rahmen von Straßenverkehrsunfällen ist z. B. die sog. DashboardInjury häufig. Der Mechanismus ist hier durch das in $90^{\circ}$ flektierte Kniegelenk mit Krafteinleitung entlang der Femurschaftachse bedingt. Häufige Frakturtypen sind vordere Pfeiler-/vordere Wandfrakturen, die Subluxation des Hüftkopfs nach anteromedial, Trümmerzonen des supraazetabulären Doms, eine impaktierte Hinterwand oder eine Mit- 


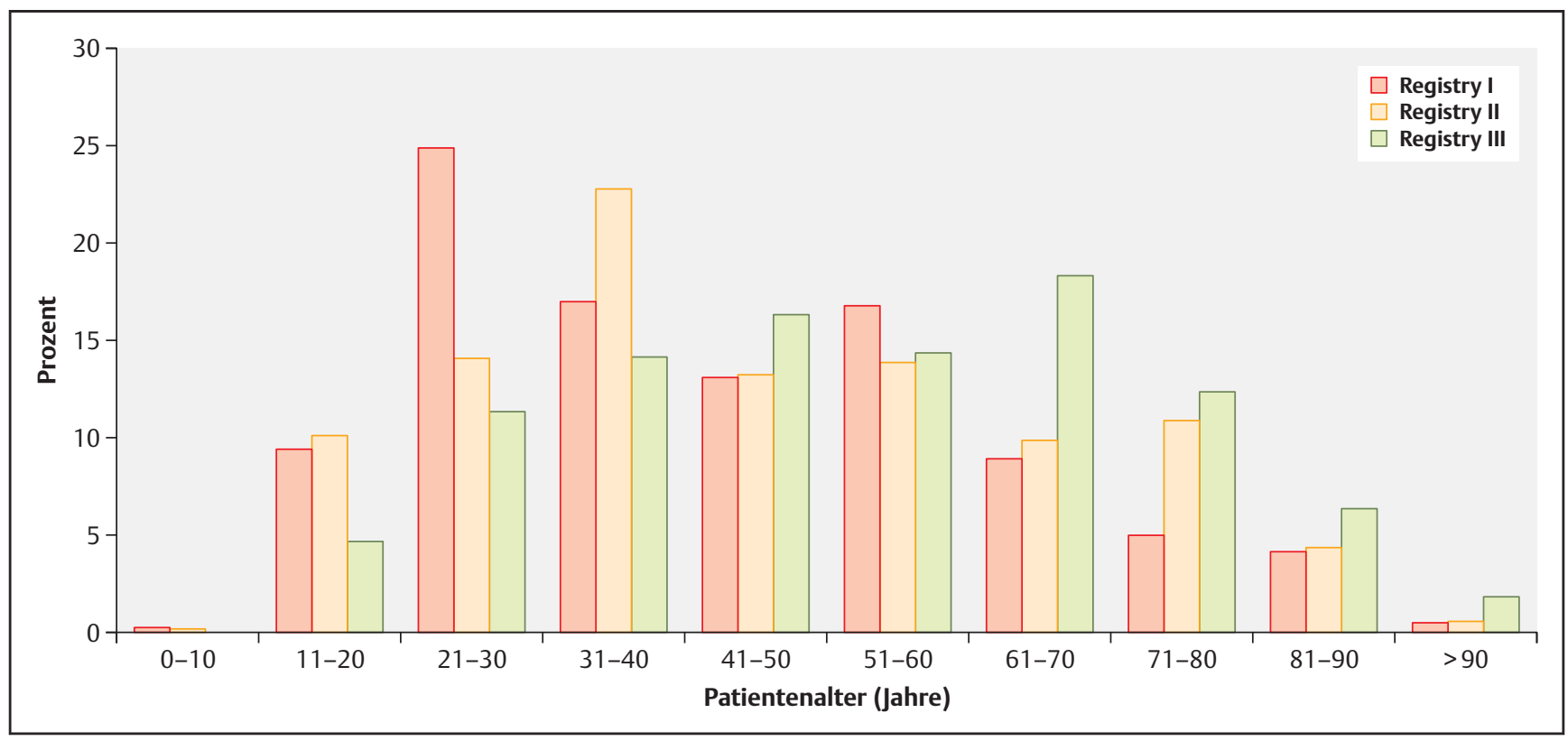

Abb. 1 Demografische Entwicklung von Azetabulumfrakturen.

beteiligung der quadrilateralen Fläche [10,11].

\section{Begleitverletzungen bei Azetabulumfraktur}

In 50\% der Fälle liegt bei Azetabulumfrakturen ein Polytrauma vor, in einem Drittel der Fälle besteht eine zusätzliche Beckenringverletzung und insgesamt weisen bis zu 75\% der Fälle Begleitverletzungen auf [2]. Diese bestimmen maßgeblich das therapeutische Prozedere und Outcome.

Begleitverletzungen, Schwere der Verletzung und die Reposition bestimmten das Outcome von Azetabulumfrakturen.

Begleitverletzungen können Femurkopffrakturen (Einteilung nach Pipkin [12]), intraartikuläre Fragmente mit konsekutiven mechanisch bedingten Knorpelschäden, Impression der azetabulären Gelenkfläche (sog. Gull-Sign im Röntgen [10]), Impression des Hüftkopfs und/ oder Kapsel-, Gefäß- und Nervenverletzungen sein, die jeweils durch eine additiv zur Fraktur bestehende Luxation des Hüftkopfs aggraviert werden können.

\section{Klassifikationen}

Klassischerweise erfolgt die Einteilung von Azetabulumfrakturen auf der anatomischen Grundlage der 2-Pfeiler-Anatomie nach Judet und Letournel [13]. Im klinischen Alltag sind v.a. die Typen 1, 10 und $8 \mathrm{zu}$ finden [13]. Es werden fol- genden Verletzungstypen unterschieden:

\section{Grundtypen:}

- Typ 1: Fraktur hinterer Pfannenrand mit/ohne dorsale Hüftkopfluxation

- Typ 2: Abrissfraktur dorsaler Pfeiler mit Dislokation

- Typ 3: Abbruch des ventralen Pfannenrands

- Typ 4: Abbruch des ventralen Pfeilers mit Dislokation, ventromediale Hüftkopfluxation

- Typ 5: Querfrakturen durch das Azetabulum, intaktes Pfannendach

Kombinationsverletzungen:

- Typ 6: T-Frakturen, Hüftkopf medialisiert

- Typ 7: Fraktur hinterer Pfeiler + hinterer Pfannenrand

- Typ 8: Querfraktur mit hinterem Pfannenrandbruch

- Typ 9: Abriss ventraler Pfeiler mit hinterem Querbruch

- Typ 10: 2-Pfeiler-Fraktur

Helfet et al. entwickelten auf Grundlage der o.g. Klassifikation das AO-Klassifikationsschema für Azetabulumfrakturen: Bei A-Frakturen ist eine Säule isoliert betroffen (partiell artikulär), bei B-Frakturen liegt eine Querkomponente vor und bei C-Frakturen sind beide Säulen und das Os ilium betroffen (komplett intraartikulär). Die von Müller et al. 1990 publizierte universelle „Comprehensive Classification of Fractures“ (CCF $=$ AO/OTA) unterteilt entsprechend hierarchisch in 3er-Gruppen (s.o.: Stabile A-Verletzungen, rotationsinstabile B-Verletzungen und translationsinstabile C-Verletzungen), die wiederum in 3 Frakturgruppen mit 3 Subgruppen unterteilt sind und durch Qualifikatoren weiter präzisiert werden.

Koxarthrosen werden unabhängig von der Ätiologie röntgenologisch nach Kellgren und Lawrence bez. ihres Schweregrads in 4 Stadien eingeteilt $[14,15]$ :

- Stadium 1: geringe subchondrale Sklerosierung, keine Osteophyten, keine Gelenkspaltverschmälerung

- Stadium 2: geringe Gelenkspaltverschmälerung, beginnende Osteophytenbildung, angedeutete Unregelmäßigkeit der Gelenkfläche

- Stadium 3: ausgeprägte Osteophytenbildung, unregelmäßige Gelenkfläche

- Stadium 4: ausgeprägte Gelenkspaltverschmälerung bis zur vollständigen Destruktion der Gelenkpartner

\section{Posttraumatische Koxarthrose}

Die häufigste Folge einer Azetabulumfraktur ist das posttraumatisch veränderte Hüftgelenk [7]: 12-57\% der Unfallverletzten entwickeln nach Azetabulumfraktur eine behandlungsbedürftige posttraumatische Koxarthrose [7,16, 17]. Die anatomische Reposition einer Azetabulumfraktur samt Wahl des geeigneten Zugangs mit entsprechender Sicht auf die dislozierten Fragmente und die intraoperative Bildgebungskontrolle der Schraubenlage sind daher wichtige Elemente in der initialen Frakturversorgung. Gelenkstufen $>2 \mathrm{~mm}$ erhöhen das 
Risiko einer sekundären Arthrose deutlich [18] und die intraartikuläre Schraubenlage bedingt ebenfalls eine rasche Gelenkzerstörung. Die Pathogenese der Arthrose bei stattgehabter undislozierter Azetabulumfraktur ist jedoch noch nicht geklärt, und in 20-30\% der Fälle tritt eine posttraumtische Koxarthrose auch bei guter Frakturreposition auf [19]. Bei dorsaler Luxation des Hüftkopfs kommt es häufig zu einer avaskulären Femurkopfnekrose, die wiederum eine sekundäre Koxarthrose bedingen kann. Weitere Ursachen sind die initiale Verletzung, übersehene oder unbehandelte osteochondrale Defekte oder weitere Komplikationen [20-22]. Die klinischen Symptome einer posttraumatischen Koxarthrose - wie z.B. Anlauf- und Ruheschmerzen oder Bewegungseinschränkung - entsprechen denen einer primären Koxarthrose. Die Therapieoption der Wahl ist nach Ausschöpfen des konservativen Therapiespektrums die Implantation einer i.d.R. zementfreien H-TEP $[19,23]$. Hierbei nimmt die Zeit zwischen Verletzung und Implantation einer H-TEP mit steigendem Alter $a b$ [19]. Unabhängig von der initialen Verletzungsschwere ist die Prothesenversorgung aber ab der 4. Lebensdekade innerhalb von 2 Jahren zu erwarten; d.h. die Zeiträume zwischen Frakturbehandlung und Prothesenversorgung scheinen altersabhängig zu sein [19] (Abb. 2).

Unabhängig von der initialen Verletzungsschwere nimmt die Zeit zwischen Fraktur und Implantation einer H-TEP mit steigendem Alter $a b$.

\section{Diagnostik}

Patienten, bei denen der Verdacht auf eine Azetabulumfraktur besteht, sind meist polytraumatisierte Patienten, die dem Schockraumalgorithmus entsprechend Diagnostik und Therapie zugeführt werden. Hier steht die Sicherung der Vitalparameter im Vordergrund, da eine Azetabulumfraktur i.d.R. keine Akutverletzung darstellt. Anamnestisch sind der Unfallhergang zu eruieren und eine klinische Untersuchung durchzuführen (Beckenkompressionsschmerz, Stellung des betroffenen Beines, periphere Durchblutung/Motorik/Sensibilität). Des Weiteren ist eine Röntgenbildgebung (Beckenübersicht, Ala- und Obturatoraufnahmen) sowie eine CTDiagnostik mit 3-D-Rekonstruktion unerlässlich [24] (Abb. 3).

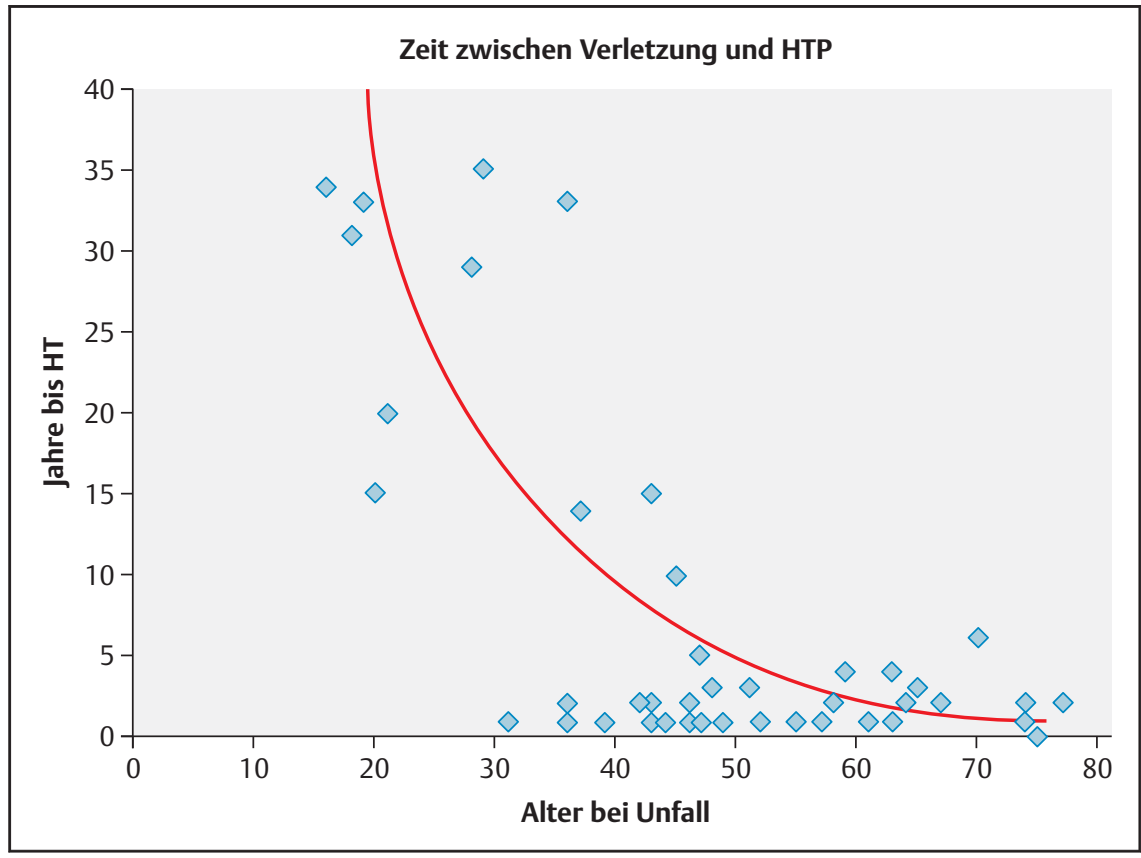

Abb. 2 Zeit zwischen Azetabulumfraktur und H-TEP-Implantation in Korrelation mit dem Patientenalter.

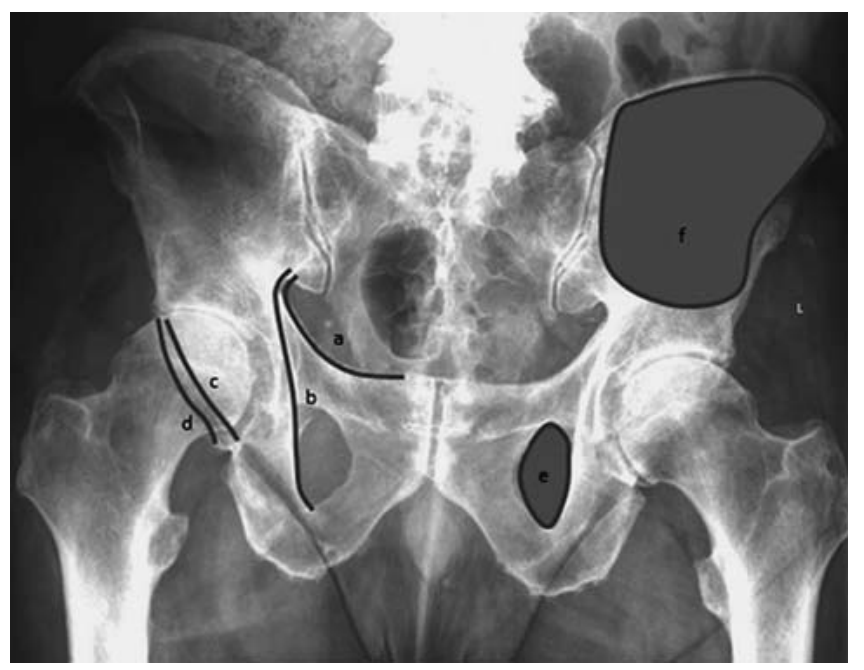

Abb. 3 Beckenübersicht mit Kennlinien ( $a$ = Linea iliopectinea, $b=$ Linea ilioischiadica, $\mathrm{C}=$ vorderer Pfannenrand, $\mathrm{d}=$ hinterer Pfannenrand, $\mathrm{e}=$ Foramen obturatorium, $\mathrm{f}=\mathrm{Ala}$ ossis ilii; hier bei vorliegender Beckenfraktur).

In der Röntgenbildgebung kann neben den o.g. Kennlinien auch das sog. RoofArc-Segment bzw. die Unversehrtheit des Pfannendachsegments beurteilt werden.

Nach stattgehabter Azetabulumfraktur und entsprechend operativer (oder auch konservativer) Versorgung umfasst die endoprothetische Diagnostik ebenfalls eine ausführliche Anamnese und klinische Untersuchung (ROM, Beinlängendifferenz, Muskulatur). Des Weiteren sollte auch immer eine Sichtung alter OP-Berichte erfolgen und die bisher erfolgte Therapie adäquat aufgearbeitet werden. In der obligaten o.g. Röntgenund CT-Untersuchung müssen die Im- plantatlage bestimmt, etwaige Knochendefekte und/oder Pseudarthrose evaluiert werden und auch die Lagebeziehungen heterotoper Ossifikationen zu Leitungsbahnen erfasst werden; ggf. kann auch eine angiografische CT-Untersuchung durchgeführt werden [25]. Bei der Durchführung einer CT sollte bei einliegendem Osteosynthesen-/Prothesenmaterial eine Anpassung der kV-Zahl zur Supprimierung von Metallartefakten erfolgen.

Röntgen- und CT-Diagnostik sind in der Frakturendoprothetik des Azetabulums obligat. 
Tab. 1 Therapieoptionen bei Azetabulumfraktur.

\begin{tabular}{|c|c|}
\hline Therapieoption & Literatur \\
\hline $\begin{array}{l}\text { ORIF } \\
\text { Open Reduc- } \\
\text { tion and Inter- } \\
\text { nal Fixation }\end{array}$ & $\begin{array}{l}\text { Helfet et al., } 1992 \text { [60] } \\
\text { Matta, } 1996 \text { [17] } \\
\text { Mears et al., 2003 [26] }\end{array}$ \\
\hline $\begin{array}{l}\text { MIOS } \\
\text { Minimally In- } \\
\text { vasive Open } \\
\text { Surgery }\end{array}$ & $\begin{array}{l}\text { Gary et al., } 2012 \text { [61] } \\
\text { Jeffcoat et al., } 2012 \text { [62] }\end{array}$ \\
\hline primäre H-TEP & $\begin{array}{l}\text { Mears et al., } 2002 \text { [63] } \\
\text { Cochu et al., } 2007 \text { [35] } \\
\text { Jauregui et al., } 2015 \text { [30] }\end{array}$ \\
\hline $\begin{array}{l}\text { Osteosynthese } \\
+ \text { primäre } \\
\text { H-TEP }\end{array}$ & $\begin{array}{l}\text { Herscovici et al., } 2010 \\
\text { [64] } \\
\text { Saxer et al., } 2011 \text { [53] } \\
\text { Lin et al., } 2015 \text { [52] }\end{array}$ \\
\hline
\end{tabular}

In Fällen unklarer Nervenläsionen kann auch eine präoperative elektrophysiologische Untersuchung sinnvoll sein. Bei entsprechender Anamnese oder vorhandenen heterotopen Ossifikationen sollte eine präoperative Bestrahlung am besten für den Tag des Eingriffs geplant werden.

Im Falle heterotoper Ossifikationen sollte eine präoperative Bestrahlung erfolgen.

\section{Therapie und Prognose}

Die Therapie und Prognose von Azetabulumfrakturen, die häufig im Zusammenhang mit einem Polytrauma auftreten, hängen u.a. vom Verletzungsausmaß und den Begleitverletzungen ab [2, 3].

Weitere prognostische Parameter sind der Frakturtyp, die Reposition und das Patientenalter [26-28]. Bei jungen Patienten mit gutem Knochenstock wird i.d.R. eine offene Reposition mit interner Fixation (ORIF) durchgeführt, wohingegen bei älteren Patienten mit schlechterer Knochenqualität neben einer ORIF auch minimalinvasive Optionen (MIOS) infrage kommen bzw. die Implantation einer Hüfttotalendoprothese ggf. in Kombination mit einer Osteosynthese zu erwägen ist (Tab.1). Die möglichen Zugänge zur operativen Versorgung von Azetabulumfrakturen sind der KocherLangenbeck-Zugang (hintere Wand, hinterer Pfeiler, Querkomponente), die chirurgische Hüftluxation nach Ganz, der ilioinguinale Zugang nach Letournel (vorderer Pfeiler, Vorderwand, Quer- komponente), der iliofemorale Zugang nach Letournel bzw. Smith-Peterson (Maryland-Modifikation; vorderer Pfeiler, Vorderwand, Femurkopf) sowie der modifizierte Stoppa-Zugang (alternativ zum ilioinguinalen Zugang) oder Pararektus-Zugang nach Keel.

Eine konservative Therapie - z. B. beim multimorbiden Patienten, bei unverschobenen Frakturen oder bei wenig dislozierten Pfannenrandfragmenten - umfasst eine Teilbelastung des betroffenen Beines für 6-12 Wochen mit 15-20 kg [29].

Eine Extensionsbehandlung kommt notfallmäßig bei Hüftkopfluxation nach dorsal infrage [29].

Neben der o.g. Option der primären Endoprothetik (ggf. in Kombination mit Osteosynthese) bei Azetabulumfrakturen umfasst die endoprothetische Versorgung auch den Fall der sekundären Endoprothetik - aufgrund einer im Verlauf auftretenden posttraumatischen Koxarthrose oder in ca. $10 \%$ der Fälle auftretenden Hüftkopfnekrose nach operativer Frakturversorgung der Azetabulumfraktur - sowie die verzögerte Primärendoprothetik. Diese bedeutet eine H-TEPImplantation im Intervall z. B. zur Knochenstockkonsolidierung nach minimalinvasiver Schraubenosteosynthese oder bei Beschwerdehaftigkeit und/oder Materialversagen bis zu 3 Monate postoperativ mit zeitnahem Verfahrenswechsel auf eine H-TEP (Tab. 2).

Die Frakturendoprothetik des Azetabulums unterscheidet sekundäre Endoprothetik (bei sekundärer Koxarthrose), primäre und verzögerte primäre Endoprothetik.

Hierbei sind insgesamt 2 Patientengruppen zu unterscheiden: Junge Patienten mit guter Knochensubstanz, die i.d.R. eine hochrasanztraumabedingte Azetabulumfraktur erleiden, und ältere Patienten (>60 Jahre), die sich mit einer mehr oder minder ausgeprägten Osteoporose und/oder Koxarthrose eine Azetabulumfraktur als typische Altersfraktur nach Sturzereignis zuziehen.

Die Ziele der endoprothetischen Versorgung sind:

- Primärstabilität der Implantate

- Wiederherstellung des anatomischen Rotationszentrums und des physiologischen Offsets [19]
- Rekonstruktion von Knochendefekten bzw. des Implantatlagers

- Langzeitstabilität durch Osteointegration

- gutes funktionelles Ergebnis

Die (verzögerte) primäre Endoprothetik im Fall einer Azetabulumfraktur betrifft ein eingeschränktes Patientenkollektiv: Patienten >65 Jahre mit einer ausgeprägten intraartikulären Frakturbeteiligung, Impaktion des azetabulären Domes einhergehend mit Osteoporose oder vorbestehender Arthrose [30] aber ggf. auch junge Patienten mit Hüftkopf- und Azetabulumbeteiligung ohne Möglichkeit einer adäquaten anatomischen Rekonstruktion [31]. Die Hauptgruppe in der Frakturendoprothetik des Azetabulums macht die sekundäre Frakturendoprothetik aus.

Die endoprothetische Versorgung nach stattgehabter Azetabulumfraktur bedarf im Allgemeinen einer umfassenden präoperativen Planung, da eine ggf. veränderte Anatomie, knöcherne Inkongruenzen, verminderte Knochenqualität bzw. Inaktivitätsdystrophie, Knochensubstanzdefekte, ggf. noch einliegendes Osteosynthesematerial oder eine insuffizient verheilte Fraktur adressiert werden müssen [20,32]. Die Voraussetzungen für eine Prothesenimplantation sind aufgrund dessen insgesamt schlechter als bei primärer Koxarthrose [20,33,34]. Aufgrund der i.d.R. anspruchsvollen Operation ist ein OP-Team mit entsprechender Expertise erforderlich.

Die endoprothetische Versorgung nach Azetabulumfraktur muss umfassend präoperativ geplant werden.

Das funktionelle Ergebnis nach HTP bei posttraumatischer Koxarthrose ist hierbei abhängig von der initialen Verletzungsschwere, wobei ein Funktionsgewinn in allen Altersklassen zu erwarten ist [19]. Das Operationsrisiko ist mit dem eines Wechsel- bzw. Revisionseingriffs vergleichbar [19].

Die endoprothetische Versorgung nach Azetabulumfrakturen stellt im sekundären Fall i.d.R. die Implantation einer zementfreien HTP, d.h. Press-fit-Pfanne, dar [23]. Im Primärfall ist aufgrund der Defektsituation bzw. vorbestehender Arthrose meist eine Abstützschale mit Bone Graft erforderlich [35]. Die Zugangswahl hängt hierbei von dem bereits verwendeten Zugang ab bzw. sollte der Lokalisation der Pathologie entspre- 
Tab. 2 Vergleich Frakturendoprothetik des Azetabulums.

\begin{tabular}{|c|c|c|c|}
\hline & primäre H-TEP bei Azetabulumfraktur & sekundäre H-TEP bei Azetabulumfraktur & verzögerte primäre H-TEP \\
\hline $\begin{array}{l}\text { Indika- } \\
\text { tionen }\end{array}$ & $\begin{array}{l}\text { - irreversible Zerstörung des Azetabu- } \\
\text { lums } \\
\text { - ausgeprägte Osteoporose } \\
\text { - Pipkin-IV-Frakturen } \\
\text { - Azetabulumfraktur bei vorbestehen- } \\
\text { der Koxarthrose }\end{array}$ & $\begin{array}{l}\text { nach vorausgegangener operativer Versor- } \\
\text { gung bei: } \\
\text { - posttraumatischer Koxarthrose } \\
\text { - Hüftkopfnekrose } \\
\text { - Pseudarthrose des Azetabulums }\end{array}$ & $\begin{array}{l}\text { - knöcherne Stabilisierung des Azeta- } \\
\text { bulums im Intervall von 8-12 Wo- } \\
\text { chen } \\
\text { - Materialversagen oder Beschwerde- } \\
\text { persistenz zeitnah nach stattgehab- } \\
\text { ter osteosynthetischer Versorgung }\end{array}$ \\
\hline Probleme & $\begin{array}{l}\text { - frühzeitige Pfannenlockerung } \\
\text { - Pseudarthrose des Azetabulums }\end{array}$ & $\begin{array}{l}\text { - einliegende Implantate } \\
\text { - okkulte Infektion } \\
\text { - heterotope Ossifikationen } \\
\text { - Narbenbildung } \\
\text { - Pseudarthrose des Azetabulums } \\
\text { - veränderte Anatomie/schwierigere Ope- } \\
\text { ration } \\
\text { - frühzeitige Pfannenlockerung }\end{array}$ & $\begin{array}{l}\text { - einliegende Implantate } \\
\text { - okkulte Infektion } \\
\text { - heterotope Ossifikationen } \\
\text { - Narbenbildung } \\
\text { - Pseudarthrose des Azetabulums } \\
\text { - veränderte Anatomie/schwierigere } \\
\text { Operation } \\
\text { - frühzeitige Pfannenlockerung }\end{array}$ \\
\hline Vorteile & $\begin{array}{l}\text { - Spongiosa aus dem eigenen Hüftkopf } \\
\text { - nur } 1 \text { OP } \\
\text { - Pfannenkomponente = Platte und } \\
\text { Pfanne } \\
\text { - ggf. gleich Belastungsstabilität }\end{array}$ & $\begin{array}{l}\text { - Versuch der Osteosynthese bzw. Hinaus- } \\
\text { zögern der Endoprothese } \\
\text { - Spongiosa aus dem eigenen Hüftkopf }\end{array}$ & $\begin{array}{l}\text { - Repositionsergebnis untergeordnet } \\
\text { - Spongiosa aus dem eigenen Hüft- } \\
\text { kopf }\end{array}$ \\
\hline
\end{tabular}

chend gewählt werden. Eine (additive) Osteosynthese wird i.d.R. mittels Beckenrekonstruktionsplatten und Schrauben durchgeführt.

Besteht der Zustand nach Osteosynthese, sollte bei geplanter Prothesenimplantation im Falle einer notwendigen Metallentfernung, um eine Irritation mit der Hüftpfanne zu vermeiden, ein zweizeitiges Vorgehen erwogen werden. Neben einer intraoperativen Abstrichentnahme aus dem Hüftgelenk bzw. dem Implantatlager sollte auch eine Sonikation des Explantats erfolgen, um mögliche okkulte Infektionen $\mathrm{zu}$ erfassen und ggf. eine nachfolgende periprothetische Infektion zu vermeiden [36].

Bestehen Osteolysen und Knochendefekte, muss eine entsprechende Rekonstruktion derselben erfolgen: Contained-Defekte sollten mit Knochen aufgefüllt werden (Auto- oder Allograft mittels Impaction Grafting [37]) und UncontainedDefekte wie auch Defekte in der Hauptbelastungszone mittels o.g. Bone Grafts und/oder Augmenten (z.B. aus Trabecular Metal ${ }^{\mathrm{TM}}$ ) adressiert werden. Die Tragfähigkeit des verbliebenen periazetabulären Knochens bestimmt ergo die Wahl des Rekonstruktionsverfahrens: Der Contained-Defekt erlaubt bei äquatorialer Kraftübertragung die Implantation hemisphärischer Press-fit-Pfannen, bei segmentalen Defekten hingegen ist die Verwendung von Revisionsstützschalen und Bone Grafts zur Erzielung einer stabilen Implantatfixation erforderlich [38].
Die verschiedenen Therapieoptionen orientieren sich hierbei an der Einteilung azetabulärer Defekte nach Paprosky et al. $[39,40]$.

Zusammengefasst ergeben sich folgende Therapieoptionen in der Frakturendoprothetik des Azetabulums:

Zugänge (ggf. in Kombination):

- Becken:

- dorsal (Kocher-Langenbeck)

- ilioinguinal (Letournel, 3 Fenster)

- intrapelvin (modifizierter StoppaZugang, Pararektus-Zugang nach Keel)

- Hüfte:

- lateral/transgluteal (Bauer)

- anterolateral (Watson-Jones)

- posterior/dorsal (Smith-Peterson)

Implantate:

- Osteosynthese:

- Reko-Platten und Schrauben

- Endoprothese:

- sphärische Press-fit-Pfanne, ggf. Pfahlschrauben, ggf. Jumbocup

- ovaläres Press-fit-Implantat mit Kranialzapfen

- Stützschalen zur Defektüberbrückung (z.B. Ganz-Schale oder Burch-Schneider-Ring), ggf. mit Augmenten in Cage-and-AugmentTechnik [41]

- Trabecular-Metal'TM-Azetabulumrevisionssystem, ggf. in Cup-andAugment-Technik [42] mit entsprechenden Trabecular-Metal-Augmenten

- additiv Bone Graft (Auto- oder Allograft; Impaction Grafting)

- individueller Beckenteilersatz

\section{Komplikationen und Langzeitergebnisse}

Neben allgemeinen postoperativen Komplikationen sind im Rahmen der Azetabulumchirurgie neben iatrogenen Gefäß- und Nervenschädigungen (A. circumflexa femoris medialis, $N$. ischiadicus) v.a. Thrombembolien durch die anfängliche Immobilisation als Komplikationsmöglichkeiten aufzuführen. Des Weiteren kann es trotz optimaler Versorgung zu einer nicht zufriedenstellenden Osteosynthese kommen. Der zunehmende Einsatz weichteilschonender Zugänge erhöht das Risiko einer suboptimalen Plattenpositionierung oder unzureichenden Fragmentreposition. Intraoperativ sollte daher immer eine computertomografische Kontrolle mittels 3-DBildwandler durchgeführt werden. Des Weiteren kann es zu einer Non-Union der Frakturfragmente nach Osteosynthese kommen sowie zu heterotopen Ossifikationen. Diese sind multifaktoriell, jedoch mit dorsalen Zugängen zum Azetabulum, Weichteilschädigungen im Allgemeinen, T-Frakturen, einer späten Frakturversorgung, einem Alter über 30 Jahren, einem additiv vorhandenen Schädel-Hirn-Trauma oder einem hohen ISS (Injury Severity Score) assoziiert [4345]. Im klinischen Alltag wird meist eine Ossifikationsprophylaxe, z. B. mittels Indometacin, durchgeführt [46]; neue Studien jedoch zeigen keinen relevanten Effekt $[47,48]$. Es bedarf diesbezüglich weiterer Evidenz. 
Tab. 3 Ergebnisse nach primärer Frakturendoprothetik des Azetabulums

\begin{tabular}{lll} 
Ergebnisse & Osteosynthese und H-TEP & Abstützring und Bone Graft \\
\hline Fallzahl (Patienten) & $6-33$ & $7-16$ \\
\hline Alter (Jahre) & $\varnothing 66-86$ & $\varnothing 70-76$ \\
\hline Follow-up (Jahre) & $0,5-5,6$ & $1,5-3,0$ \\
\hline Harris Hip Score (Punkte) & $69-88$ & $74-85$ \\
\hline Komplikationsrate (\%) & $15-41$ & $29-31$ \\
\hline Pfannenlockerung (\%) & 5-9 & $0-8$ \\
\hline Literatur & Lin et al., 2015 [52] & Cochu et al., 2007 [35] \\
& Saxer et al., 2011 [53] & Simko et al., 2006 [66] \\
& Herscovici et al., 2010 [64] & Tidermark et al., 2003 [67] \\
Boraiah et al., 2009 [65] & Hoellen et al., 1997 [68]
\end{tabular}

Tab. 4 Ergebnisse nach sekundärer Frakturendoprothetik des Azetabulums.

\begin{tabular}{lll}
\hline Ergebnisse & $\begin{array}{l}\text { zementfreie/Press-fit- } \\
\text { Pfannenverankerung }\end{array}$ & $\begin{array}{l}\text { zementierte Pfannenver- } \\
\text { ankerung und Bone Graft }\end{array}$ \\
\hline Fallzahl (Patienten) & $18-53$ & 20 \\
\hline Alter (Jahre) & $\varnothing 42-53$ & $\varnothing 53$ \\
\hline Follow-up (Jahre) & $3,3-8,4$ & $9,5-18$ \\
\hline Harris Hip Score (Punkte) & $77-90$ & $82-93$ \\
\hline Komplikationsrate (\%) & $15-18$ & $10-25$ \\
\hline Pfannenlockerung (\%) & 2-8 & $10-15$ \\
\hline Literatur & Lizaur-Utrilla et al., 2012 [23] & $\begin{array}{l}\text { Bronsema et al., 2014 [72] } \\
\text { Schreurs et al., 2005 [34] }\end{array}$ \\
\hline & $\begin{array}{l}\text { Zhang et al., 2011 [69] } \\
\text { Lai et al., 2011 [70] }\end{array}$ & \\
& $\begin{array}{l}\text { Chu et al., 2010 [71] } \\
\text { Pavelka et al., 2006 [51] } \\
\text { Bellabarba et al., 2001 [32] }\end{array}$ & \\
&
\end{tabular}

Allgemeine Komplikationsmöglichkeiten in der Hüftendoprothetik sind Protheseninfektionen, aseptische oder septische Lockerung, (rezidivierende) Luxationen und zunehmend auch periprothetische Frakturen des Femurs und des Azetabulums.

Die Ergebnisse der H-TEP-Implantation nach Azetabulumfraktur sind schlechter als nach primärer H-TEP-Implantation bei primärer Koxarthrose und mit den Ergebnissen nach Revisions- und Wechseleingriffen vergleichbar [32,33,49-51]. Die allgemeine Komplikationsrate in der sekundären Frakturendoprothetik des Azetabulums beträgt durchschnittlich $20 \%[19,32,33]$ und erhöht sich in der primären Frakturendoprothetik auf bis zu $40 \%[35,52,53]$. Selten finden sich Ergebnisse, die mit der Primärendoprothetik vergleichbar sind [32,54]. Vergleicht man die Ergebnisse sekundärer Endoprothetik mit den Daten der H-TEP-Implantation bei primärer Koxarthrose, zeigen sich eine verlängerte Operationszeit $[23,32]$, ein erhöhtes Risiko für heterotope Ossifikationen [55] und Infektionen [20] sowie ein vermehrter Blutverlust und Transfusionsbedarf [32]. Bei jüngeren Patienten ist eine kürzere Standzeit beschrieben einhergehend mit einem höheren Komplikations- und Infektrisiko [56]. Es handelt sich hierbei um aseptische Lockerungen der Prothesenkomponenten bei zementierten Pfannen und infolge starken Abriebs bei funktionell anspruchsvollen, jungen und aktiven Patienten.

Die Ergebnisse der Endoprothetik bei Azetabulumfraktur gleichen meist denen der Revisionsendoprothetik.

Aktuelle Studien zeigen sowohl niedrigere Überlebensraten und erhöhte Komplikationsraten [57] als auch gute mittlere bis langfristige Ergebnisse nach $\mathrm{H}$-TEP-Implantation bei sekundärer $\mathrm{Ar}$ throse oder Hüftkopfnekrose mit verbesserter Funktion und guter Standzeit [58].
Funktionszugewinne sind in allen Altersklassen zu erwarten [19]. Für das funktionelle Ergebnis sind die initiale Frakturschwere und die Frakturbehandlung relevant [19].

Bei adäquater Indikationsstellung für die primäre Implantation einer $\mathrm{H}$-TEP bei Azetabulumfraktur lassen sich bez. Überleben, Funktion und Komplikationsrate gute Ergebnisse erzielen [30,59]. Auch die Daten für die Kombination aus ORIF und H-TEP sind zufriedenstellend [52].

Insgesamt bedarf die Evidenz bez. der Frakturendoprothetik noch weiterer Studien [59] (Tab. 3 und 4).

\section{Fallbeispiele}

\section{Fallbeispiel 1: primäre Endoprothetik}

Die 87-jährige Patientin zog sich bei einem einfachen Sturzereignis eine dislozierte Azetabulumfraktur links zu (vordere Pfeilerfraktur mit hintere Hemiquerkomponente mit Protrusion des Hüftkopfs ins kleine Becken; CCF A3.3a3c3d3e4; Abb.4). Zunächst wurde eine suprakondyläre Extension angelegt. Nach ausführlicher präoperativer Vorbereitung bei kardialer und onkologischer Multimorbidität erfolgte die Versorgung mittels Schneider-Burch-Ring, einer passenden PE-Pfanne und einem zementfreien Standardschaft (Abb. 5).

\section{Fallbeispiel 2a: verzögerte primäre Endoprothetik}

Bei dem 51-jährigen Patienten bestand der Zustand nach Polytrauma nach Hochrasanztrauma u.a. mit traumatischer dorsaler Hüftgelenkluxation mit Zertrümmerung des hinteren Pfeilers und des Pfannendachs rechts, einer Fraktur des Ramus ossis pubis inferior rechts, einer Femurschaftfraktur links und einem hämorrhagischen Schock bei Blutung aus der A. glutealis superior rechts. Auswärts wurde u.a. neben der kardiopulmonalen Stabilisierung eine offene Reposition und Plattenosteosynthese des Azetabulums rechts 7 Tage nach Trauma durchgeführt (Abb.6 und 7). Der Patient wurde uns zur Therapieoptimierung bei in hinterer Luxation stehender Hüfte und Trümmerzone im Bereich des Azetabulums bzw. des Hüftkopfs zuverlegt. Diesbezüglich erfolgte 1 Monat nach Trauma eine Revision des rechten Hüftgelenks mit Resektion von Vernarbungen und heterotopen Ossifikationen sowie mit Implantation eines Schnei- 


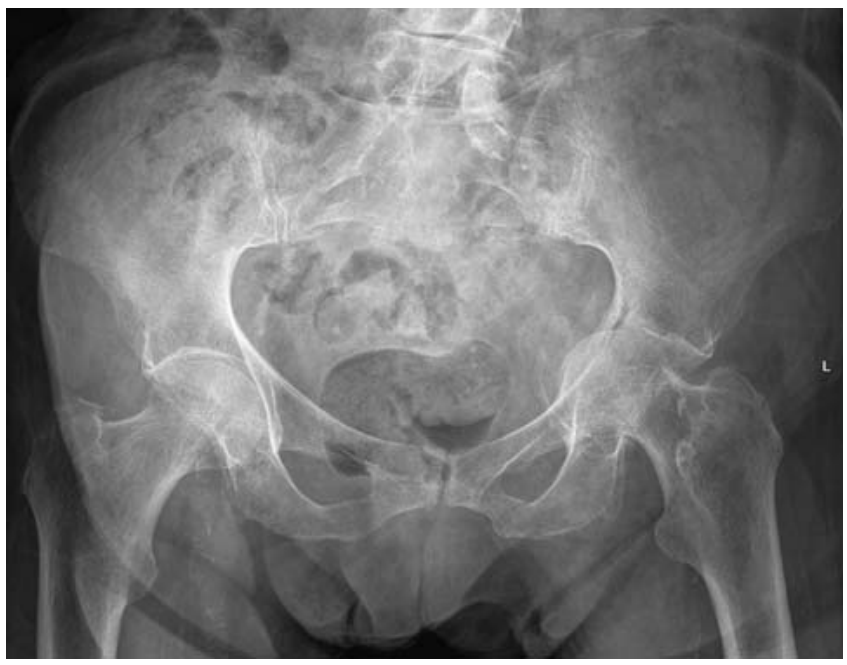

Abb.4 Beckenübersicht, präoperativ: dislozierte Azetabulumfraktur links.

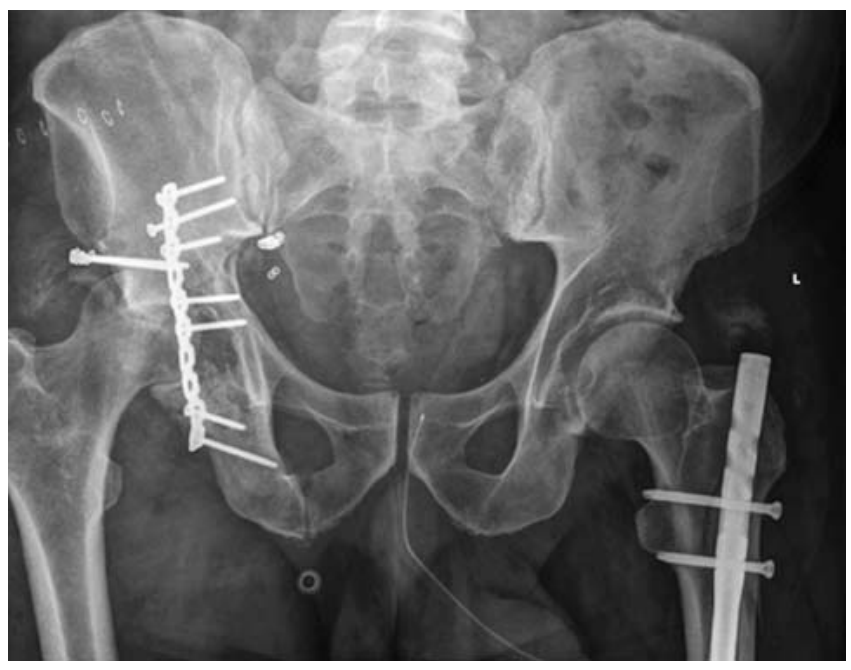

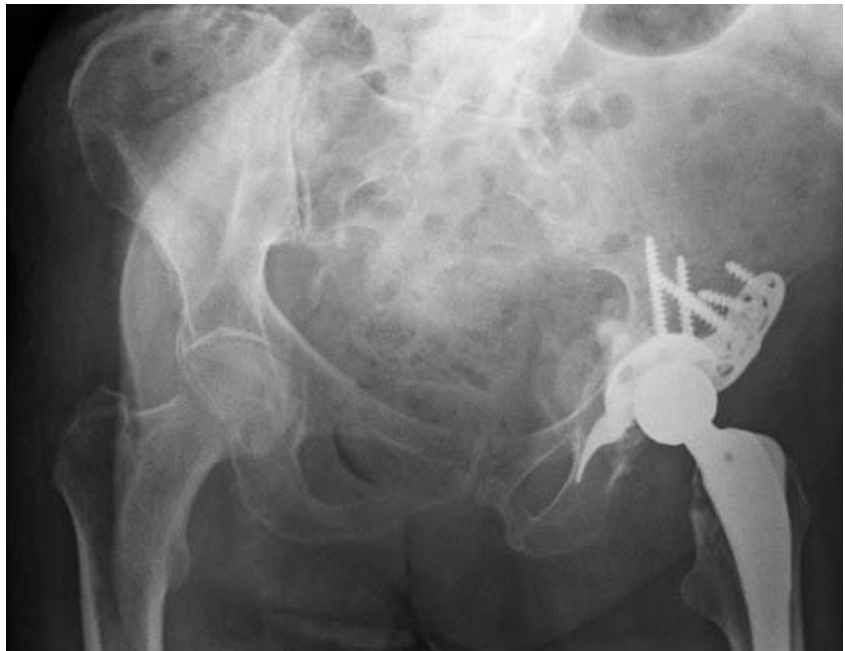

Abb. 5 Beckenübersicht, postoperativ: Versorgung mittels Abstützring und autologer Spongiosaplastik.

Abb. 6 Beckenübersicht 8 Wochen nach osteosynthetischer Versorgung mit Subluxation des Hüftkopfs und ausgeprägter Defektsituation des Hüftkopfs und der hinteren Wand.

der-Burch-Abstützrings zur Rekonstruktion des Hüftgelenkzentrums, einer Hinterwandrekonstruktion aus einem Spongiosablock des Hüftkopfs, einer Pfannenbodenplastik mit allogenen und autogenen Spongiosachips sowie die Implantation einer zementfreien H-TEP rechts (Abb. 8). In den weiteren Verlaufskontrollen zeigte sich eine diskrete $\mathrm{Zu}$ nahme der heterotopen Ossifikationen (Brooker Grad III). Der Patient ist jedoch relativ beschwerdearm und kommt im Alltag mit einer relativen Bewegungseinschränkung gut zurecht.

\section{Fallbeispiel $2 b$ : verzögerte primäre Endoprothetik}

Bei dem 55-jährigen Patienten bestand eine Hüftkopfnekrose rechts 3 Monate nach Plattenosteosynthese des vorderen und hinteren Pfeilers bei komplexer Azetabulumfraktur im Rahmen einer Polytraumatisierung ex domo mit Inkongru- enz im Bereich der Gelenkpfanne, ausbleibender knöcherner Konsolidierung und Defektsituationen im Bereich des hinteren Pfeilers, der hinteren Wand und der quadrilateralen Fläche (Paprosky Typ III A; Abb. 9 und 10).

In Vorbereitung auf die H-TEP-Implantation erfolgte zunächst die Revision des rechten Hüftgelenks über das 1 . Fenster des ilioinguinalen Zugangs mit Teilmetallentfernung sowie Abstrich- und Gewebeprobenentnahme für die histopathologische Diagnostik und Sonikation des Explantats. Die Ergebnisse waren negativ. Es erfolgte dann über den Kocher-Langenbeck-Zugang die Revision des Hüftgelenks mit Implantation einer zementfreien H-TEP rechts, mit Implantation einer modularen Pfannenschale mit Iliumlasche und Kranialzapfen zur Erzielung einer proximalen Krafteinleitung, eine allogene und autologe Spongiosaplastik im Bereich des Pfannen-

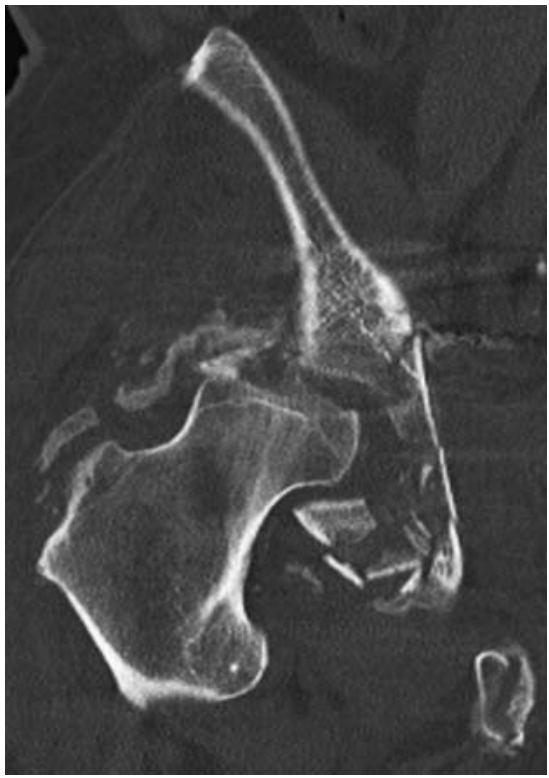

Abb. 7 CT Hüfte rechts nativ: Subluxationsfehlstellung und Trümmerzone am Azetabulum und Hüftkopf.

grunds und der Azetabulumhinterwand sowie die Implantation einer additiven Platte zur Stabilisierung des hinteren Pfeilers nach Entfernung der einliegenden Plattenosteosynthese (Abb. 11).

\section{Fallbeispiel 3: sekundäre Endoprothetik}

Der 51-jährige Patient stellte sich in unserer Sprechstunde mit posttraumatischer Koxarthrose links mit Beschwerdehaftigkeit seit 1 Jahr und ausgeprägten heterotopen Ossifikationen vor. Er hatte sich vor 26 Jahren eine Azetabulumfraktur links zugezogen, die auswärts ilioinguinal und über den Kocher-Langenbeck-Zugang mit einer Plattenosteosynthese versorgt wurde (Abb. 12 und 13). 


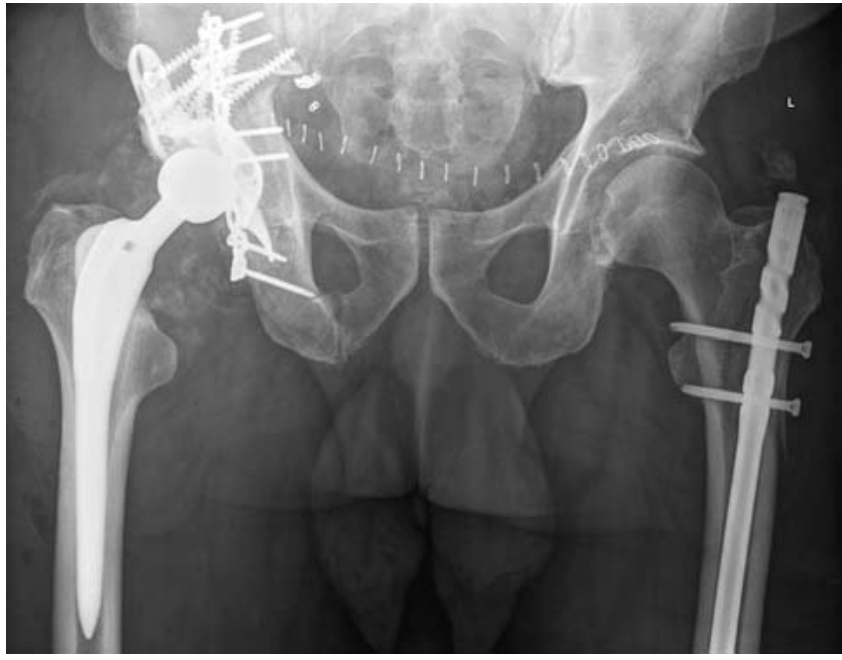

Abb. 8 Beckenübersicht, 3 Monate postoperativ: Schneider-BurchRing und heterotope Ossifikationen (Brooker Grad III).

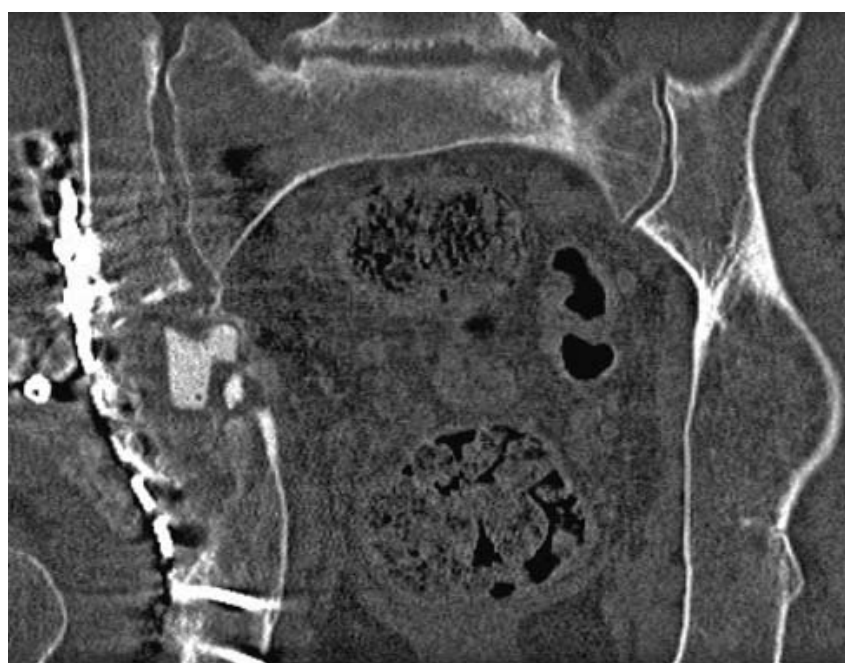

Abb.10 CT Becken mit Metallartefaktesupprimierung: Defektsituation der quadrilateralen Fläche.

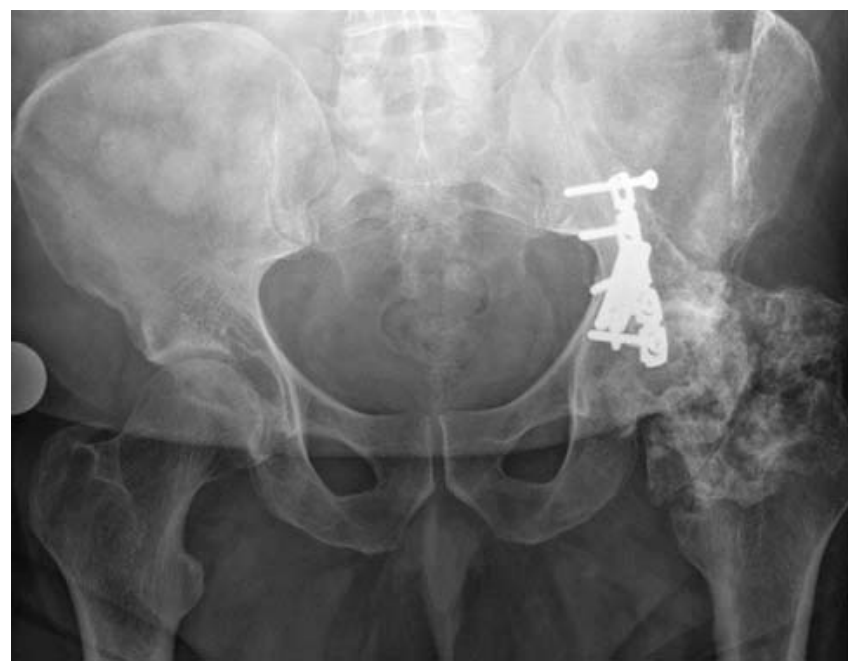

Abb. 12 Beckenübersicht nach langjährig zurückliegender azetabulärer Plattenosteosynthese links mit sekundärer/posttraumatischer Koxarthrose links.

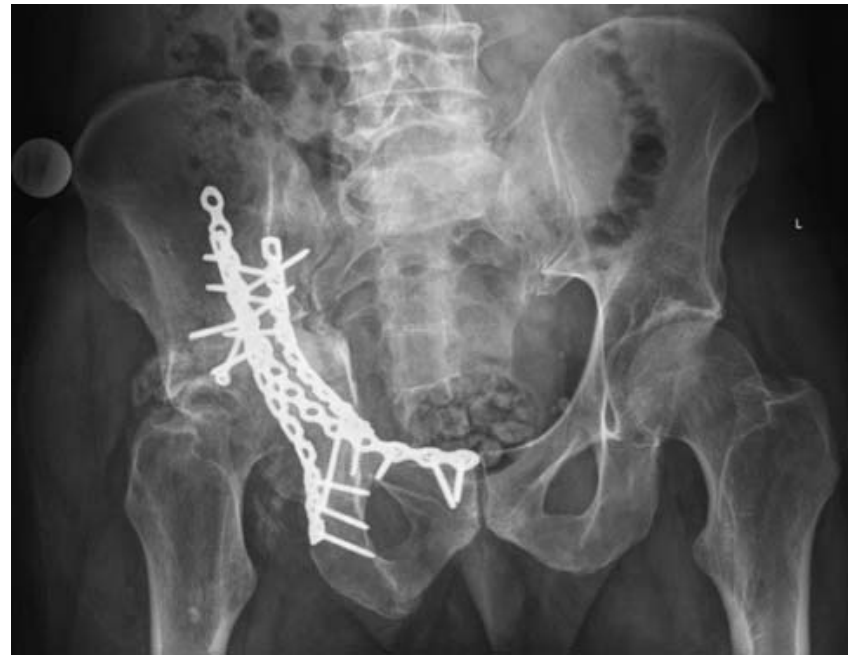

Abb. 9 Beckenübersicht 6 Wochen nach Osteosynthese beider Pfeiler mit Inkongruenz im supraazetabulären Dom sowie ausgeprägter Defektzone im Bereich der quadrilateralen Fläche und des Hüftkopfs.

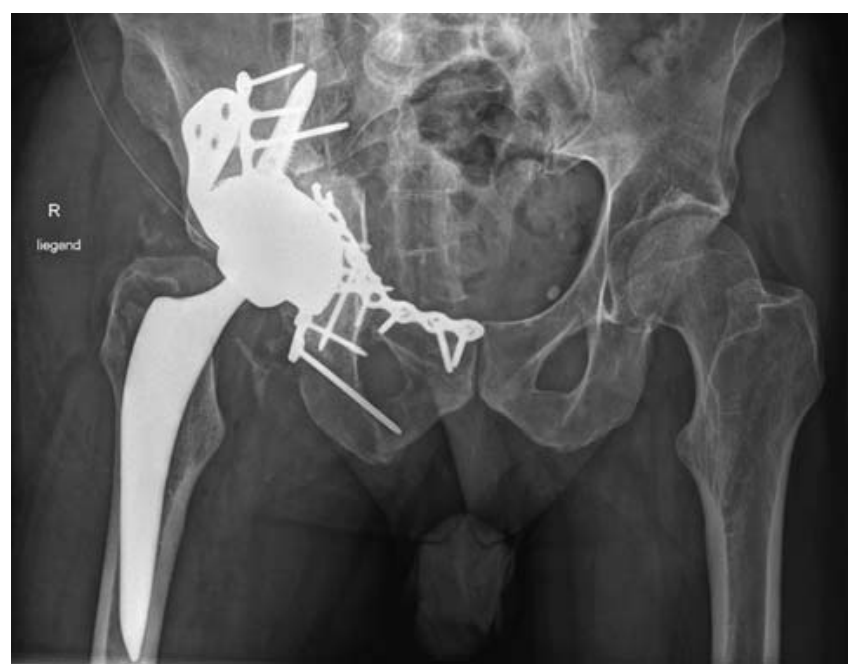

Abb. 11 Beckenübersicht, postoperativ: modulare Pfannenschale mit Iliumlasche und Kranialzapfen sowie Reosteosynthese des hinteren Pfeilers.

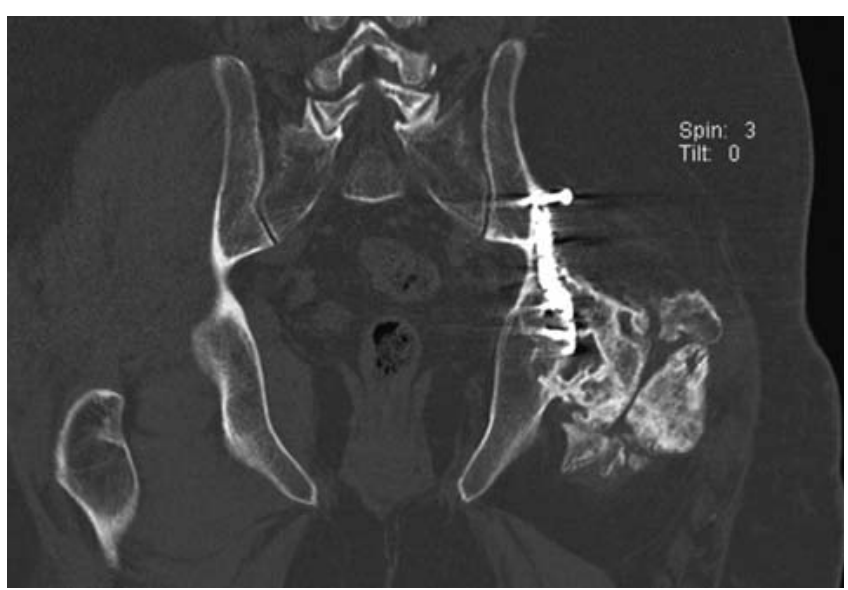

Abb.13 CT Becken nativ mit Metallartefaktsupprimierung: ausgeprägte heterotope Ossifikationen. 


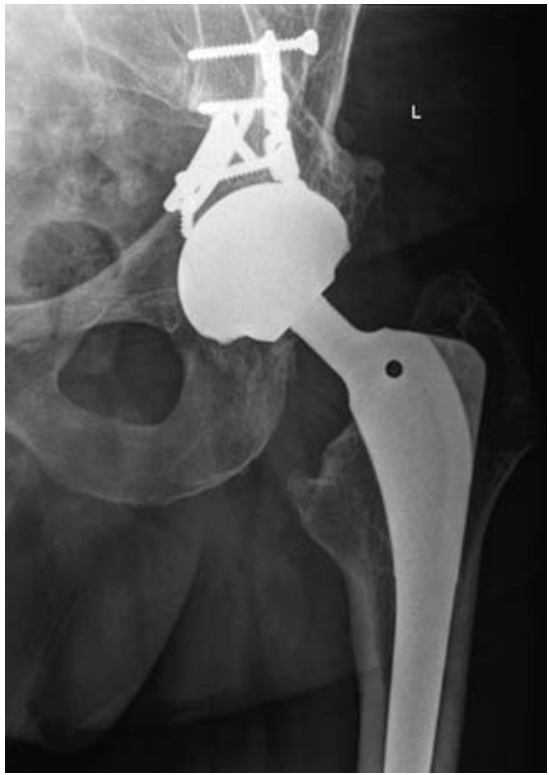

Abb. 14 Hüfte links a.-p., postoperativ: sekundäre zementfreie $\mathrm{H}$-TEP-Implantation.

Nach präoperativer Bestrahlung erfolgte über den transglutealen Zugang nach Bauer mit Ausnutzung des unteren Narbenverlaufs nach Kocher-Langenbeck eine Arthrolyse, die Resektion von heterotopen Ossifikationen, eine Teilmetallentfernung, eine Spongiosplastik am proximalen Femur, eine transossäre Refixation der Glutealmuskulatur sowie die Implantation einer zementfreien H-TEP (Abb. 14). In den Verlaufskontrollen zeigt sich der Patient zufrieden. Eine rückläufige Schraube einer der Platten zeigt sich im Vergleich zu den Voraufnahmen unverändert.

\section{Schlussfolgerung}

Die Frakturendoprothetik des Azetabulums ist hinsichtlich der Indikationsstellung wie auch der chirurgischen Therapie herausfordernd und anspruchsvoll. Es handelt sich oft um individuelle Indikationsstellungen und Therapieplanungen. Die Komplikationsraten und Langzeitergebnisse sind im Durchschnitt mit den Daten der Wechsel- bzw. Revisionsendoprothetik vergleichbar, aktuelle Studien weisen jedoch bei adäquater Indikationsstellung gute Ergebnisse auf. Das Patientenkollektiv der Frakturendoprothetik des Azetabulums ist klein; bei einer jedoch zunehmend älter werdenden Gesellschaft - einhergehend mit Osteoporose, Arthrose und weiteren Begleiterkrankungen - aber auch bei einem wachsenden Anteil an aktiven und anspruchsvollen älteren Patienten ist eine Relevanzzunahme zu erwarten. Die Versorgung aller Patientenkollektive sollte am Zentrum erfolgen und von einem Team durchgeführt werden, das sowohl Erfahrung in der Azetabulumchirurgie als auch Revisionsendoprothetik des Hüftgelenks hat.

\section{Interessenkonflikt: Nein.}

\section{Literatur}

${ }^{1}$ Laird A, Keating JF. Acetabular fractures: a 16year prospective epidemiological study. The Journal of bone and joint surgery British volume 2005; 87: 969-973

2 Rüter A, Trentz O, Wagner M. Unfallchirurgie. München: Elsevier; 2003

3 Kregor PJ, Templeman D. Associated injuries complicating the management of acetabular fractures: review and case studies. The Orthopedic clinics of North America 2002; 33: 73-95, viii

${ }^{4} \mathrm{Kim} J W$, Herbert B, Hao J et al. Acetabular fractures in elderly patients: a comparative study of low-energy versus high-energy injuries. International orthopaedics 2015; 39: 11751179

${ }^{5}$ Winkler KH. Facharzt Orthopädie. München: Elsevier; 2011

${ }^{6}$ Ochs BG, Marintschev I, Hoyer H et al. Changes in the treatment of acetabular fractures over 15 years: analysis of 1266 cases treated by the German Pelvic Multicentre Study Group (DAO/DGU). Injury 2010; 41: 839-851

Giannoudis PV, Grotz MR, Papakostidis C et al. Operative treatment of displaced fractures of the acetabulum. A meta-analysis. The Journal of bone and joint surgery British volume 2005; 87: 2-9

${ }^{8}$ Brown TD, Johnston RC, Saltzman CL et al. Posttraumatic osteoarthritis: a first estimate of incidence, prevalence, and burden of disease. Journal of orthopaedic trauma 2006; 20: 739-744

9 Culemann U, Tosounidis G, Pohlemann T. [Fractures of the accetabulum-treatment strategies and actual diagnostics]. Zentralbl Chir 2005; 130: W58-W71

10 Anglen JO, Burd TA, Hendricks KJ et al. The "Gull Sign": a harbinger of failure for internal fixation of geriatric acetabular fractures. Journal of orthopaedic trauma 2003; 17 : 625-634

${ }^{11}$ Ferguson TA, Patel $R$, Bhandari $M$ et al. Fractures of the acetabulum in patients aged 60 years and older: an epidemiological and radiological study. The Journal of bone and joint surgery British volume 2010; 92: 250257

12 Thannheimer A, Gutsfeld P, Bühren V. Aktuelle Therapieoptionen bei Hüftkopfluxationsfrakturen. Chirurg 2009; 80: 1140-1146

13 Letournel E. Acetabulum fractures: classification and management. Clinical orthopaedics and related research 1980: 81-106

${ }^{14}$ Kohn MD, Sassoon AA, Fernando ND. Classifications in Brief: Kellgren-Lawrence Classification of Osteoarthritis. Clinical orthopaedics and related research 2016; 474: 1886-1893

${ }^{15}$ Kellgren JH, Lawrence JS. Radiological assessment of osteo-arthrosis. Annals of the rheumatic diseases 1957; 16: 494-502

${ }^{16}$ Mears DC, Velyvis JH. Primary total hip arthroplasty after acetabular fracture. Instr Course Lect 2001; 50: 335-354

${ }^{17}$ Matta JM. Fractures of the acetabulum: accuracy of reduction and clinical results in patients managed operatively within three weeks after the injury. The Journal of bone and joint surgery American volume 1996; 78: 1632-1645

${ }^{18}$ Kreder HJ, Rozen N, Borkhoff CM et al. Determinants of functional outcome after simple and complex acetabular fractures involving the posterior wall. The Journal of bone and joint surgery British volume 2006; 88: 776782

${ }^{19}$ Frank C, Siozos P, Wentzensen A et al. [Total hip replacement for coxarthrosis following acetabular fracture. Significance of age and injury severity]. Der Unfallchirurg 2010; 113: 1013-1018, 1020-1012

${ }^{20}$ Ranawat A, Zelken J, Helfet $D$ et al. Total hip arthroplasty for posttraumatic arthritis after acetabular fracture. The Journal of arthroplasty $2009 ; 24: 759-767$

21 Weber M, Berry DJ, Harmsen WS. Total hip arthroplasty after operative treatment of an acetabular fracture. The Journal of bone and joint surgery American volume 1998; 80: 1295-1305

${ }^{22}$ Wright R, Barrett K, Christie MJ et al. Acetabular fractures: long-term follow-up of open reduction and internal fixation. Journal of orthopaedic trauma 1994; 8: 397-403

${ }^{23}$ Lizaur-Utrilla A, Sanz-Reig J, Serna-Berna R. Cementless acetabular reconstruction after acetabular fracture: a prospective, matchedcohort study. The journal of trauma and acute care surgery 2012; 73: 232-238

${ }^{24}$ Kaulbach C, Heller M, Triebel HJ et al. [Radiologic diagnosis of acetabular fractures]. Der Radiologe 1989; 29: 501-507

${ }^{25}$ Simon P, von Roth P, Perka C. Treatment algorithm of acetabular periprosthetic fractures. International orthopaedics 2015; 39: 1995 2003

${ }^{26}$ Mears DC, Velyvis JH, Chang CP. Displaced acetabular fractures managed operatively: indicators of outcome. Clinical orthopaedics and related research 2003; 407: 173-186

${ }^{27}$ Moed BR, WillsonCarr SE, Watson JT. Results of operative treatment of fractures of the posterior wall of the acetabulum. The Journal of bone and joint surgery American volume 2002; 84-A: 752-758

${ }^{28}$ Murphy D, Kaliszer M, Rice J et al. Outcome after acetabular fracture. Prognostic factors and their inter-relationships. Injury 2003; 34: $512-517$

${ }^{29}$ Morrigl B. Becken. Stuttgart: Thieme; 2014

30 Jauregui JJ, Clayton A, Kapadia BH et al. Total hip arthroplasty for acute acetabular fractures: a review of the literature. Expert Rev Med Devices 2015; 12: 287-295

31 Sierra RJ, Mabry TM, Sems SA et al. Acetabular fractures: the role of total hip replacement. The bone \& joint journal 2013; 95-B: 11-16

${ }^{32}$ Bellabarba C, Berger RA, Bentley $C D$ et al. Cementless acetabular reconstruction after acetabular fracture. The Journal of bone and joint surgery American volume 2001; 83-A: 868-876

33 Berry DJ, Halasy M. Uncemented acetabular components for arthritis after acetabular fracture. Clinical orthopaedics and related research 2002; 22: 164-167

34 Schreurs BW, Zengerink M, Welten ML et al. Bone impaction grafting and a cemented cup after acetabular fracture at 3-18 years. Clinical orthopaedics and related research 2005; 437: 145-151

35 Cochu G, Mabit C, Gougam Tet al. [Total hip arthroplasty for treatment of acute acetabular fracture in elderly patients]. Revue de chirurgie orthopedique et reparatrice de l'appareil moteur 2007; 93: 818-827

${ }^{36}$ Klatte TO, Sabihi R, Guenther D et al. High rates of occult infection after shoulder fracture fixation: considerations for conversion 
shoulder arthroplasty. HSS journal: the musculoskeletal journal of Hospital for Special Surgery 2015; 11: 198-203

37 Slooff TJ, Buma P, Schreurs BW et al. Acetabular and femoral reconstruction with impacted graft and cement. Clinical orthopaedics and related research 1996: 108-115

38 Gravius S, Pagenstert G, Weber $O$ et al. Azetabuläre Defektrekonstruktion in der Revisionschirurgie der Hüfte. Orthopäde 2009; 38: 729-740

39 Paprosky WG, Perona PG, Lawrence JM. Acetabular defect classification and surgical reconstruction in revision arthroplasty. A 6 year follow-up evaluation. The Journal of arthroplasty 1994; 9: 33-44

40 Paprosky WG, O'Rourke M, Sporer SM. The treatment of acetabular bone defects with an associated pelvic discontinuity. Clinical orthopaedics and related research 2005; 441: 216-220

${ }^{41}$ Gunther KP, Wegner T, Kirschner S et al. [Modular reconstruction in acetabular revision with antiprotrusio cages and metal augments: the cage-and-augment system]. Operative Orthopadie und Traumatologie 2014 26: $141-155$

42 Makinen TJ, Kuzyk P, Safir OA et al. Role of Cages in Revision Arthroplasty of the Acetabulum. The Journal of bone and joint surgery American volume 2016; 98: 233-242

${ }^{43}$ Ghalambor N, Matta JM, Bernstein L. Heterotopic ossification following operative treatment of acetabular fracture. An analysis of risk factors. Clinical orthopaedics and related research 1994; 305: 96-105

${ }^{44}$ Daum WJ, Scarborough MT, Gordon W jr. et al. Heterotopic ossification and other perioperative complications of acetabular fractures. Journal of orthopaedic trauma 1992; 6: 427432

45 Elhassan Y, Abdelhaq A, Piggott RP et al. Heterotopic Ossification following acetabular fixation: incidence and risk factors: 10-year experience of a tertiary centre. Injury 2016 47: 1332-1336

${ }^{46}$ Burd TA, Lowry KJ, Anglen JO. Indomethacin compared with localized irradiation for the prevention of heterotopic ossification following surgical treatment of acetabular fractures. The Journal of bone and joint surgery American volume 2001; 83-A: 1783-1788

47 Rashid RH, Qadir I, Ahmed Wet al. Prophylaxis against heterotopic ossification after elbow and acetabular fractures - Do we really need it? JPMA The Journal of the Pakistan Medical Association 2015; 65: S87-S90

48 Griffin SM, Sims SH, Karunakar MA et al. Heterotopic ossification rates after acetabular fracture surgery are unchanged without indomethacin prophylaxis. Clinical orthopaedics and related research 2013; 471: 2776 2782

49 Glas PY, Bejui-Hugues J, Carret JP. [Total hip arthroplasty after treatment of acetabular fracture]. Revue de chirurgie orthopedique et reparatrice de l'appareil moteur 2005; 91 : 124-131

${ }^{50}$ Matta JM, Ferguson TA. Total hip replacement after acetabular fracture. Orthopedics 2005 28: 959-960
51 Pavelka T, Linhart M, Houcek P. [Hip joint arthroplasty following surgical treatment of acetabular fracture]. Acta chirurgiae orthopaedicae et traumatologiae Cechoslovaca 2006; 73: $268-274$

${ }^{52}$ Lin C, Caron J, Schmidt AH et al. Functional outcomes after total hip arthroplasty for the acute management of acetabular fractures: 1- to 14-year follow-up. Journal of orthopaedic trauma 2015; 29: 151-159

${ }^{53}$ Saxer F, Studer P, Jakob M. [Open stabilization and primary hip arthroplasty in geriatric patients with acetabular fractures: combination of minimally invasive techniques]. Unfallchirurg 2011; 114: 1122-1127

54 Boardman KP, Charnley J. Low-friction arthroplasty after fracture-dislocations of the hip. The Journal of bone and joint surgery British volume 1978: 60-B: 495-497

55 Iorio $R$, Healy WL. Heterotopic ossification after hip and knee arthroplasty: risk factors, prevention, and treatment. The Journal of the American Academy of Orthopaedic Surgeons 2002; 10: 409-416

${ }^{56}$ Berger RA, Jacobs JJ, Quigley LR et al. Primary cementless acetabular reconstruction in patients younger than 50 years old. 7- to 11year results. Clinical orthopaedics and related research 1997: 216-226

57 Mont MA, Elmallah RK. CORR Insights((R)): Total hip arthroplasty after acetabular fracture is associated with lower survivorship and more complications. Clinical orthopaedics and related research 2016; 474: 399401

$58 \mathrm{Yu}$ L, Zhang $\mathrm{CH}$, Guo T et al. [Middle and longterm results of total hip arthroplasties for secondary post-traumatic arthritis and femoral head necrosis after acetabular fractures]. Zhongguo gu shang = China journal of orthopaedics and traumatology 2016; 29: 109113

${ }^{59}$ De Bellis UG, Legnani C, Calori GM. Acute total hip replacement for acetabular fractures: a systematic review of the literature. Injury 2014; 45: 356-361

60 Helfet DL, Borrelli J jr., DiPasquale T et al. Stabilization of acetabular fractures in elderly patients. The Journal of bone and joint surgery American volume 1992; 74: 753-765

61 Gary JL, VanHal M, Gibbons SD et al. Functional outcomes in elderly patients with acetabular fractures treated with minimally invasive reduction and percutaneous fixation. Journal of orthopaedic trauma 2012; 26: 278-283

62 Jeffcoat DM, Carroll EA, Huber FG et al. Operative treatment of acetabular fractures in an older population through a limited ilioinguinal approach. Journal of orthopaedic trauma 2012; 26: 284-289

${ }^{63}$ Mears DC, Velyvis JH. Acute total hip arthroplasty for selected displaced acetabular fractures: two to twelve-year results. The Journal of bone and joint surgery American volume 2002; 84-A: 1-9

${ }^{64}$ Herscovici D jr., Lindvall E, Bolhofner B et al. The combined hip procedure: open reduction internal fixation combined with total hip arthroplasty for the management of acetabular fractures in the elderly. Journal of orthopaedic trauma 2010; 24: 291-296
${ }^{65}$ Boraiah S, Ragsdale M, Achor T et al. Open reduction internal fixation and primary total hip arthroplasty of selected acetabular fractures. Journal of orthopaedic trauma 2009; 23: $243-248$

${ }^{66}$ Simko P, Braunsteiner T, Vajczikova S. [Early primary total hip arthroplasty for acetabular fractures in elderly patients]. Acta chirurgiae orthopaedicae et traumatologiae Cechoslovaca 2006; 73: 275-282

${ }^{67}$ Tidermark J, Blomfeldt R, Ponzer S et al. Primary total hip arthroplasty with a BurchSchneider antiprotrusion cage and autologous bone grafting for acetabular fractures in elderly patients. Journal of orthopaedic trauma 2003: 17: 193-197

${ }^{68}$ Hoellen IP, Mentzel M, Bischoff $M$ et al. [Acetabular fractures in elderly persons. Primary endoprosthetic treatment]. Orthopade 1997; 26: 348-353

${ }^{69}$ Zhang L, Zhou Y, Li Y et al. Total hip arthroplasty for failed treatment of acetabular fractures: a 5-year follow-up study. The Journal of arthroplasty $2011 ; 26: 1189-1193$

${ }^{70}$ Lai O, Yang J, Shen B et al. Midterm results of uncemented acetabular reconstruction for posttraumatic arthritis secondary to acetabular fracture. The Journal of arthroplasty 2011; 26: 1008-1013

71 Chu XB, Tong PJ, Zhang JH. [Total hip arthroplasty for the post-traumatic osteoarthritis after open reduction and internal fixation of acetabular fracture]. Zhonghua wai ke za zhi [Chinese journal of surgery] 2010; 48: 1097-1100

72 Bronsema E, te Stroet MA, Zengerink $M$ et al. Impaction bone grafting and a cemented cup after acetabular fracture. International orthopaedics 2014; 38: 2441-2446

\section{Dr. med. Anna Janine Schreiner Assistenzärztin UWCH}

Dr. med. Peter Marten de Zwart Sektionsleiter Endoprothetik PD Dr. med. Fabian Maria Stuby Leitender Oberarzt, Sektionsleiter Traumatologie

Berufsgenossenschaftliche Unfallklinik Tübingen

Schnarrenbergstraße 95 72076 Tübingen

Prof. Dr. med. Björn Gunnar Ochs Sektionsleiter Endoprothetik

Department Chirurgie

Klinik für Orthopädie und

Unfallchirurgie

Universitätsklinikum Freiburg

Hugstetter Straße 55

79106 Freiburg

www.uniklinik-freiburg.de

gunnar.ochs@uniklinik-freiburg.de 